\title{
TERC Variants Associated with Short Leukocyte Telomeres: Implication of Higher Early Life Leukocyte Telomere Attrition as Assessed by the Blood-and-Muscle Model
}

\author{
Simon Toupance ${ }^{1,2} \mathbb{D}$, Maria G. Stathopoulou ${ }^{3}$, Alexandros M. Petrelis ${ }^{3}$, Vesna Gorenjak ${ }^{3}$, \\ Carlos Labat ${ }^{1}{ }^{10}$, Tsung-Po Lai ${ }^{4}$, Sophie Visvikis-Siest ${ }^{3}{ }^{(1)}$ and Athanase Benetos ${ }^{1,2, *}$ \\ 1 Université de Lorraine, Inserm, DCAC, F-54000 Nancy, France; s.toupance@chru-nancy.fr (S.T.); \\ carlos.labat@inserm.fr (C.L.) \\ 2 Université de Lorraine, CHRU-Nancy, Pôle "Maladies du Vieillissement, Gérontologie et Soins Palliatifs", \\ F-54000 Nancy, France \\ 3 Université de Lorraine, IGE-PCV, F-54000 Nancy, France; maria.stathopoulou@inserm.fr (M.G.S.); \\ apetrelis@live.com (A.M.P.); gorenjak.vesna@gmail.com (V.G.); sophie.visvikis-siest@inserm.fr (S.V.-S.) \\ 4 Center of Human Development and Aging, Rutgers, New Jersey Medical School, \\ The State University of New Jersey, Newark, NJ 07103, USA; t1599@njms.rutgers.edu \\ * Correspondence: a.benetos@chru-nancy.fr; Tel.: +33-383-15-33-22; Fax: +33-383-15-76-68
}

Received: 29 April 2020; Accepted: 27 May 2020; Published: 31 May 2020

\begin{abstract}
Short leukocyte telomere length (LTL) is associated with atherosclerotic cardiovascular disease (ASCVD). Mendelian randomisation studies, using single nucleotide polymorphisms (SNPs) associated with short LTL, infer a causal role of LTL in ASCVD. Recent results, using the blood-and-muscle model, indicate that higher early life LTL attrition, as estimated by the ratio between LTL and skeletal muscle telomere length (MTL), rather than short LTL at conception, as estimated by MTL, should be responsible of the ASCVD-LTL connection. We combined LTL and MTL measurements and SNPs profiling in 402 individuals to determine if 15 SNPs classically described as associated with short LTL at adult age were rather responsible for higher LTL attrition during early life than for shorter LTL at birth. Two of these SNPs (rs12696304 and rs10936599) were associated with LTL in our cohort ( $p=0.027$ and $p=0.025$, respectively). These SNPs, both located on the TERC gene, were associated with the LTL/MTL ratio ( $p=0.007$ and $p=0.037$, respectively), but not with MTL ( $p=0.78$ and $p=0.32$ respectively). These results suggest that SNPs located on genes coding for telomere maintenance proteins may contribute to a higher LTL attrition during the highly replicative first years of life and have an impact later on the development of ASCVD.
\end{abstract}

Keywords: telomere length maintenance; SNPs; TERC; atherosclerosis; cohort studies

\section{Introduction}

Short leukocyte telomere length (LTL) is associated with atherosclerotic cardiovascular disease (ASCVD) and its complications [1,2]. Genome-wide association studies (GWAS) have found several single nucleotide polymorphisms (SNPs) associated with short LTL over the years [3,4] and recently Mendelian randomisation studies showed that these genetic variants are associated with ASCVD [5-7] inferring a causal role of short LTL in atherosclerosis. The hypothesis is that inherited short LTL is associated with ASCVD. However, we recently showed, using the blood and muscle model, that the association between short LTL and ASCVD was not related to shorter telomere length (TL) at the beginning of life—as estimated by minimally proliferative skeletal muscle telomere length (MTL)—but 
to higher LTL attrition, especially during the first years of life, as estimated by the LTL/MTL ratio [8]. This lead us to the hypothesis that genetic variants associated with short LTL measured at adult age identified by GWAS could be related to higher LTL attrition during early life rather than to shorter LTL at conception. In this genetic study, we tested this hypothesis by combining SNPs profiling of 15 variants associated with short LTL and telomere length measurements in leukocytes and skeletal muscles. We found that SNPs associated with short LTL in our cohort, TERC variants rs12696304 and rs10936599, are linked with higher estimated early life LTL attrition rather than with shorter estimated LTL at birth.

\section{Materials and Methods}

\subsection{The Cohort}

The aim of the TELARTA (Telomere in Arterial Aging) study is to examine the implication of telomere dynamics in arterial aging and the development of atherosclerosis using the blood and muscle model. The study and its goals have been described previously [8]. Briefly, 259 men and women (older than 20 years), who were admitted for various surgical procedures, were enrolled in university hospitals in Nancy $(n=215)$ and Marseille $(n=44)$, France. A replication cohort of 143 individuals was enrolled in 3 sites: 91 individuals in original sites (Nancy and Marseille) and 52 individuals in 3 Athens hospitals (Onassis Cardiac Surgery Center, Surgeon KP; Iaso General Hospital Surgeon MVG; Hippokration Hospital, Surgeon EM). All 402 subjects gave their informed consent for inclusion before they participated in the study. The study was conducted in accordance with the Declaration of Helsinki, and the protocol was approved by Ethics Committee (Comité de Protection des Personnes) of Nancy, France, Ethics Committee of the University of Athens and Ethics Committee of each one of the 3 participating hospitals. This study is registered on http://www.clinicaltrials.gov under unique identifier: NCT02176941.

\subsection{Telomere Length Measurements}

Leukocyte TL (LTL) and Muscle TL (MTL) were measured in DNA extracted by the phenol/chloroform/isoamyl alcohol method from peripheral blood leukocytes and skeletal muscle biopsies ( $100 \mathrm{mg}$ in the surgical field), respectively. DNA samples passed an integrity testing using a $1 \%(\mathrm{wt} / \mathrm{vol})$ agarose gel before TL measurements performed by Southern blotting of the terminal restriction fragments (TRFs), as described previously [9]. Briefly, DNA samples were digested $\left(37^{\circ} \mathrm{C}\right.$ ) overnight with the restriction enzymes Hinf I and Rsa I (Roche Diagnostics GmbH, Mannheim, Germany). Digested DNA samples and DNA ladders were resolved on 0.5\% (wt/vol) agarose gels during $23 \mathrm{~h}$. DNA was depurinated, denatured, neutralised and transferred onto a positively charged nylon membrane (Roche Diagnostics $\mathrm{GmbH}$ ) using a vacuum blotter (Biorad, Hercules, CA, USA). Membranes were hybridised at $65{ }^{\circ} \mathrm{C}$ with a DIG-labelled telomeric probe after which the probe was detected by the DIG luminescent detection procedure (Roche Diagnostics $\mathrm{GmbH}$ ) and exposed on CCD camera (Las 4000, Fujifilm Life Sciences, Cambridge, MA, USA). Optical density values (OD) versus DNA migration distances (in pixels) were obtained from pictures with image processing software (Multigauge, Fujifilm Life Sciences). Distances were converted to molecular weight (MW) in kilobases $(\mathrm{kb})$ using a power function transformation owing to DNA ladders. Mean TRF length was calculated in the 3-20 kb range using the formula mean TRF $(\mathrm{kb})=\Sigma(\mathrm{ODi}) / \Sigma(\mathrm{ODi} / \mathrm{MWi})$, where ODi is the OD at position $i$ and MWi is the MW (i.e., TRF length) at that position. Leukocyte and muscle DNA from one patient were always measured simultaneously on the same membrane, as shown in the representative blot (Figure 1), and measurements were performed in duplicate on separate membranes. The measurement repeatability, as determined by the intraclass correlation coefficient, was 0.99 (95\% confidence interval, $0.817-1.0$ ) and 0.98 (95\% confidence interval, $0.81-1.0)$ for LTL and MTL, respectively. The repeatability of the means of 2 duplicates (used in the analysis), known as the 
extrapolated repeatability, was 0.995 and 0.991 for LTL and MTL, respectively. The LTL/MTL ratio was calculated.

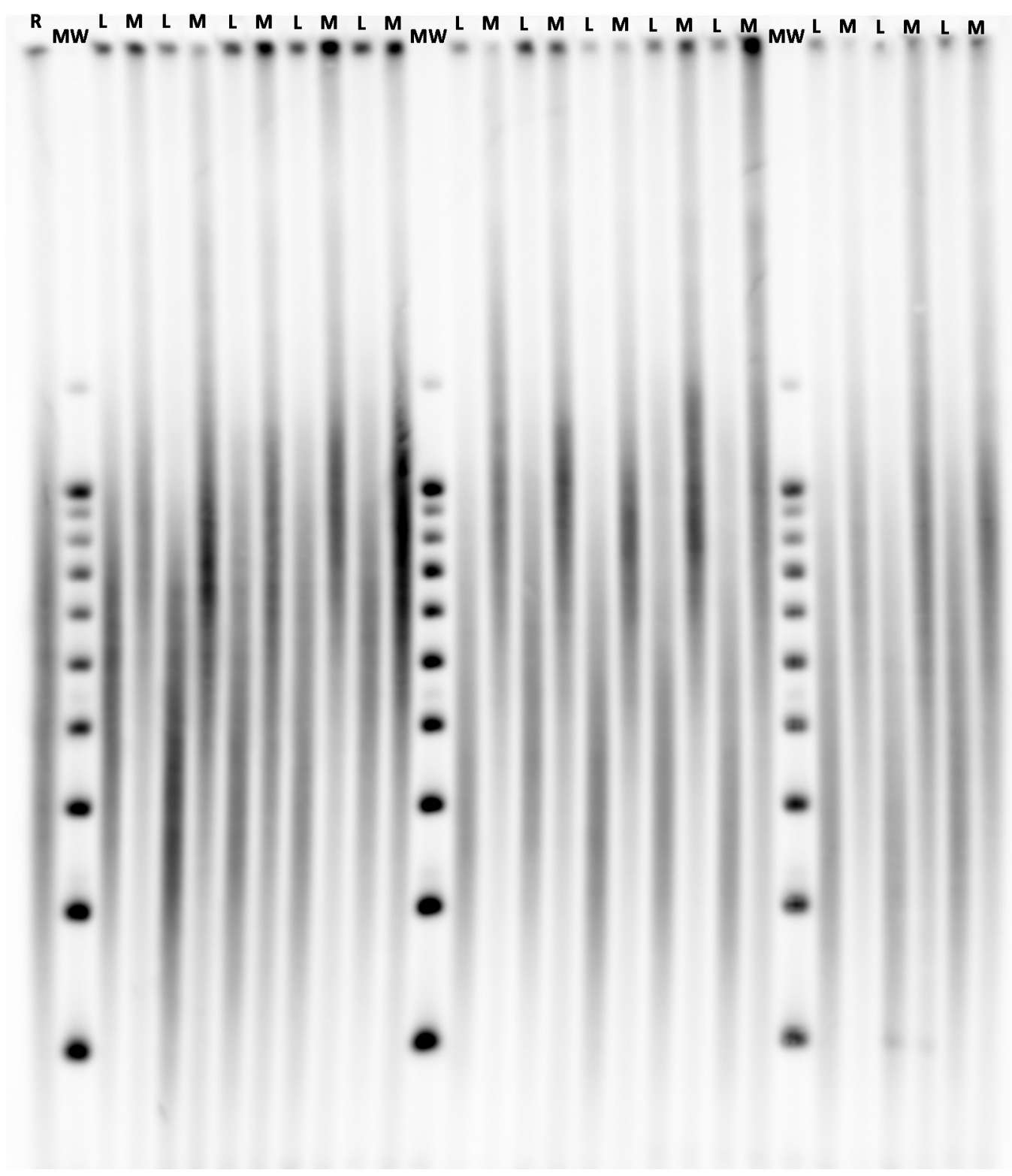

Figure 1. Illustrative membrane of telomere length measurements performed by Southern blotting of the terminal restriction fragments (TRFs). A sample of known telomere length serves as an internal reference (R). Three molecular weight ladders (MW) are resolved across the gel, and the one that is closest to a given sample is used for the computation of its mean TRF. Leukocyte (L) and Muscle (M) DNA samples of one participant are resolved on adjacent lanes.

\subsection{Single Nucleotide Polymorphism Genotyping}

Fifteen SNPs (rs11125529, rs6772228, rs12696304, rs10936599, rs7675998, rs7726159, rs2736100, rs9419958, rs9420907, rs4387287, rs3027234, rs8105767, rs412658, rs6028466 and rs755017), observed previously to be associated with LTL in several GWAS [5], were genotyped in DNA samples using a PCR-based KASP assay [10]. Minor allele frequencies (MAF) were calculated and the Hardy-Weinberg equilibrium was tested using the $\chi^{2}$ method. SNPs which did not follow Hardy-Weinberg equilibrium were excluded in the subsequent analyses. 


\subsection{Statistical Analyses}

Continuous variables are presented as means \pm SD or mean \pm SE and discrete variables as frequencies or percentages. Pairwise comparisons were performed using the Mann-Whitney and $\chi^{2}$ tests, as appropriate. Telomere length values are presented and compared with or without adjustment to age and sex. Bivariate relations between continuous variables were determined using Pearson correlation coefficients. Statistical analyses were performed using the Number Crunching Statistical System (NCSS) 9 statistical software package (NCSS, Kaysville, UT, USA).

For SNP analyses, LTL was transformed into $\log$ LTL to normalize its distribution. Statistical analyses were performed using linear regression models adjusted for age and sex for LTL and MTL, and age only for the LTL/MTL ratio (MTL and LTL but not LTL/MTL are sex-dependant). Direct SNP associations were examined with PLINK software in an additive model (using minor alleles as reference alleles) and epistatic interactions with PLINK and R softwares and the CAPE R package. Taking into account the limited sample size, and in order to limit multiple testing, the LTL phenotype was initially tested and only SNPs associated with LTL in our cohort were tested against MTL and LTL/MTL phenotypes.

\section{Results}

\subsection{Population Characteristics}

Participants were of European ancestry, aged $60 \pm 15$ years (with an age range of 20-94 years) and $32 \%$ were females. Among them, $48 \%$ displayed clinical manifestations of ASCVD in either coronary, carotid, cerebral, iliac, femoral or popliteal arteries.

\subsection{Telomere Length Dynamics}

$\mathrm{LTL}($ mean $\pm \mathrm{SD} ; 6.71 \pm 0.84 \mathrm{~kb})$ was shorter than MTL $(8.57 \pm 0.72 \mathrm{~kb} ; p<0.0001)$ in all individuals. Mean LTL/MTL ratio was $0.78 \pm 0.07$. Both LTL and MTL shortened with age with a slope of the effect of age steeper for LTL than MTL ( -31 bp/year and -15 bp/year, respectively). The LTL/MTL ratio decreased with age $(-0.0021 \pm 0.0002 /$ year $)$. Age-adjusted LTL was longer in women than men $(6.86 \pm 0.06 \mathrm{~kb}$ and $6.64 \pm 0.04 \mathrm{~kb}$, respectively; $p<0.005)$. Similarly, age-adjusted MTL was longer in women than men $(8.74 \pm 0.06 \mathrm{~kb}$ and $8.49 \pm 0.04 \mathrm{~kb}$, respectively; $p<0.001)$, whereas age-adjusted LTL/MTL was not influenced by sex $(0.78 \pm 0.005$ in women vs. $0.78 \pm 0.004$ in men; $p=0.69)$.

\subsection{Single Nucleotide Polymorphisms Association with Telomere Length}

The 13 SNPs in accordance with Hardy-Weinberg equilibrium (rs11125529, rs6772228, rs12696304, rs10936599, rs7675998, rs2736100, rs9419958, rs9420907, rs4387287, rs3027234, rs8105767, rs412658 and rs6028466) were included in the analyses. Two of these SNPs, rs12696304 and rs10936599, were significantly associated with LTL ( $p=0.027$ and $p=0.025$, respectively, Table 1$)$. These SNPs map to a locus harbouring TERC, the gene encoding the RNA subunit of telomerase. The minor alleles, allelic frequencies, and directions of the associations were similar to previous findings [5]. These two SNPs showed no association with MTL ( $p=0.78$ and $p=0.32$, respectively, Table 2 ) but were associated with the LTL/MTL ratio ( $p=0.007$ and $p=0.037$, respectively), in a way that the allele associated with shorter LTL was associated with a lower LTL/MTL ratio, thus signalling higher LTL attrition (Figure 2). No significant epistatic interaction was identified. 
Table 1. Single nucleotide polymorphisms association with leukocyte telomere length (LTL).

\begin{tabular}{cccccccccc}
\hline & & & & & & \multicolumn{3}{c}{ Log LTL } \\
\hline SNP & CHR & Position & Gene & $\begin{array}{c}\text { Effect } \\
\text { Allele }\end{array}$ & $\begin{array}{c}\text { Other } \\
\text { Allele }\end{array}$ & MAF & BETA & SE & P \\
\hline rs11125529 & 2 & 54248729 & ACYP2 & A & C & 0.12 & 0.000024 & 0.0050 & 0.99 \\
rs6772228 & 3 & 58390292 & PXK & A & T & 0.045 & -0.0094 & 0.0073 & 0.20 \\
rs12696304 & 3 & 169763483 & TERC & G & C & 0.29 & -0.0074 & 0.0033 & 0.027 \\
rs10936599 & 3 & 169774313 & TERC & T & C & 0.25 & -0.0080 & 0.0036 & 0.025 \\
rs7675998 & 4 & 163086668 & NAF1 & A & G & 0.24 & -0.0012 & 0.0037 & 0.74 \\
rs2736100 & 5 & 1286401 & TERT & A & C & 0.47 & -0.0038 & 0.0032 & 0.24 \\
rs9419958 & 10 & 103916188 & OBFC1 & T & C & 0.16 & -0.00093 & 0.0044 & 0.84 \\
rs9420907 & 10 & 103916707 & OBFC1 & C & A & 0.17 & 0.00032 & 0.0042 & 0.94 \\
rs4387287 & 10 & 103918139 & OBFC1 & A & C & 0.21 & 0.0038 & 0.0039 & 0.32 \\
rs3027234 & 17 & 8232774 & CTC1 & T & C & 0.21 & 0.0017 & 0.0038 & 0.66 \\
rs8105767 & 19 & 22032639 & ZNF208 & G & A & 0.30 & -0.0027 & 0.0034 & 0.42 \\
rs412658 & 19 & 22176638 & ZNF676 & T & C & 0.39 & -0.0016 & 0.0032 & 0.63 \\
rs6028466 & 20 & 39500359 & DHX35 & A & G & 0.070 & -0.0022 & 0.0062 & 0.72 \\
\hline
\end{tabular}

LTL = Leukocyte telomere length, SNP = Single nucleotide polymorphism, CHR = Chromosome, Position = base-pair position (GRCh38.p3), MAF = minor allelic frequency, BETA = regression coefficient (change in telomere length per copy of the effect allele), SE = standard error. LTL values are transformed in Log LTL to normalize their distribution. Analyses are age- and sex-adjusted.

Table 2. TERC single nucleotide polymorphisms (SNPs) association with muscle telomere length (MTL).

\begin{tabular}{ccccccccc}
\hline & & & & \multicolumn{4}{c}{ MTL } \\
\hline SNP & CHR & Position & Gene & $\begin{array}{c}\text { Effect } \\
\text { Allele }\end{array}$ & $\begin{array}{c}\text { Other } \\
\text { Allele }\end{array}$ & BETA & SE & P \\
\hline rs12696304 & 3 & 169763483 & TERC & G & C & -0.014 & 0.051 & 0.78 \\
rs10936599 & 3 & 169774313 & TERC & T & C & -0.054 & 0.054 & 0.32 \\
\hline
\end{tabular}

MTL = Muscle telomere length, SNP = Single nucleotide polymorphism, $\mathrm{CHR}=$ Chromosome, Position = base-pair position (GRCh38.p3), BETA = regression coefficient (change in telomere length per copy of the effect allele), $\mathrm{SE}=$ standard error. Analyses are age- and sex-adjusted.

(a)

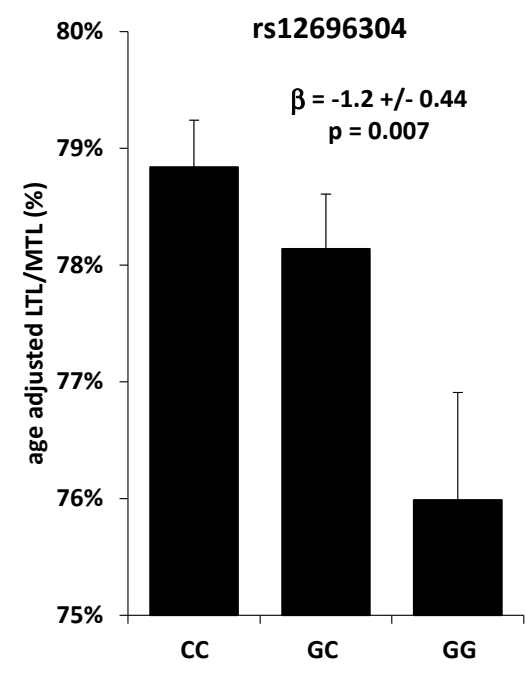

(b)

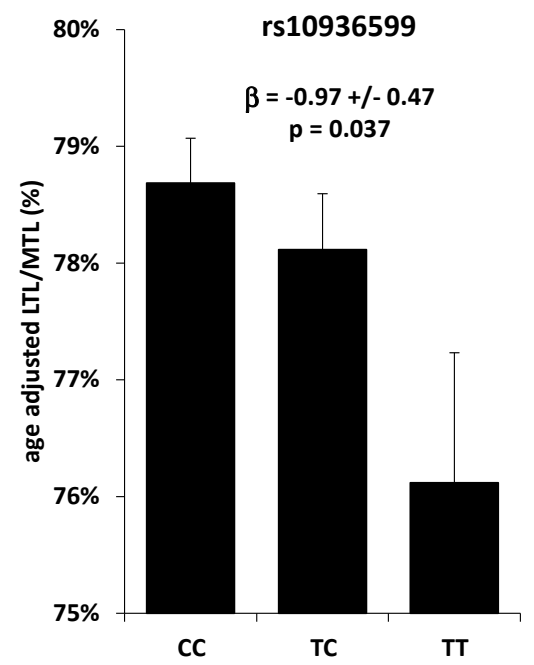

Figure 2. TERC SNPs association with LTL/MTL (a) LTL/MTL ratio (expressed in \%) according to Table 12696304. (TERC) genotype; (b) LTL/MTL ratio (expressed in \%) according to the rs10936599 (TERC) genotype. $\mathrm{LTL}=$ Leukocyte telomere length, $\mathrm{MTL}=$ Muscle telomere length, $\beta=$ change in ratio per copy of the effect allele Values are presented as mean +/- SEM. Analyses are age-adjusted. 


\section{Discussion}

In our cohort of patients, SNPs that were found to be associated with short LTL are associated with a lower LTL/MTL ratio but are not associated with MTL. The present results indicate that rs12696304 and rs10936599, two TERC SNPs previously described as associated with short LTL, do not influence TL at conception as reflected by MTL, but rather higher LTL attrition from conception onwards as expressed in the LTL/MTL ratio. We have reported previously that the gap between LTL and MTL is essentially constituted during the first decade of life [11]. SNPs located on genes coding for telomere maintenance proteins may thus contribute to higher TL attrition during this critical highly replicative period and have an impact later on the development of ASCVD [12-14].

The observed associations of these SNPs with LTL but not MTL may also suggest that person-to-person variation in TL dynamics specifically in the hematopoietic system but not in skeletal muscle, and presumably not in most other tissues, might contribute to the pathogenesis of atherosclerosis. This contribution may possibly be mediated through inflammation regulation [15]. Indeed, short telomeres have been shown to upregulate the NLRP3 (NOD (nucleotide oligomerization domain)-, LRR (leucine-rich repeat)-, and PYD (pyrin domain)-containing protein 3) inflammasome of macrophages in a murine model [16]. NLRP3, an innate immune signalling complex, is known to activate the proinflammatory IL-1 pathway, which contribute to atherogenesis [17]. Moreover, clonal haematopoiesis of indeterminate potential (CHIP), a phenomenon which is associated with a doubling of the risk of ASCVD [18,19], has been recently linked to short LTL [20,21] and the upregulation of the macrophage NLRP3 inflammasome in human [22,23]. Another possible explanation of the link observed between short LTL and ASCVD is through the hemothelium paradigm. As the hematopoietic system and vascular endothelium share a common precursor, the hemogenic endothelium or hemothelium [24], short LTL might reflect short TL in progenitor cells involved in endothelial repair. Compromised proliferation capacity of endothelial progenitor cells with short TL may influence endothelium repair capacity and impact ASCVD progression [25].

This study is limited by the small sample size in regard to SNPs analyses, but it is difficult to obtain very large number of participants due to the heavy procedure needed to obtain results for MTL. However, this drawback was offset at least for TL measurements by the use of high reproducibility TRF method [26,27].

In conclusion, taking advantage from the data obtained by the blood and muscle telomere model and those obtained from Mendelian randomisation studies, the present study proposes a new mechanical insight in the association between short LTL and ASCVD. Our findings suggest that TERC variants might impact early life TL attrition in hematopoietic cells and contribute later to the pathogenesis of atherosclerosis.

Author Contributions: S.T. and A.B. conceived and designed the study. S.T. and T.-P.L. performed the T.-P.L. measurements. M.G.S. and V.G. performed the SNP profiling. S.T., M.G.S., A.M.P. and C.L. performed the analyses. M.G.S., A.M.P., V.G. and C.L. contributed to the manuscript preparation. S.T. and A.B. wrote the manuscript with input from all co-authors. A.B. and S.V.-S. oversaw all experimental design, data analysis, and manuscript preparation. All authors have read and agreed to the published version of the manuscript.

Funding: This work was supported by the French National Research Agency (ANR), Translationnelle: $N^{\circ}$ ID RCB: 2014-A00298-39: 2014-2017, the French regional project CPER-ITM2P 2015-2020, the French PIA project « Lorraine Universite d'Excellence », reference ANR-15-IDEX-04-LUE and the Investments for the Future program under grant agreement No. ANR-15-RHU-0004.

Acknowledgments: Members of the TELARTA consortium: S. Gautier, G. Watfa, P. Lacolley, V. Regnault, H. Louis, C. Lakomy, M. Folio, M. Schmitt, A. Didier, S. Vauthier, N. François, A. Aviv, M. Kimura, P.M. Rossi, F. Dignat George, F. Sabatier, S. Simoncini, C. Dumoulin, P. Béranger, S. Malikov, N. Settembre, J. Hubert, L. Frimat, B. Bertrand, M. Boufi, X. Flecher, N. Sadoul, P. Eschwege, M. Kessler, I.P. Tzanetakou, I.P. Doulamis, P. Konstantopoulos, A. Tzani, M. Korou, A. Gkogkos, K. Perreas, E. Menenakos, G. Samanidis, M. Vasiloglou-Gkanis, J.D. Kark, S. Verhulst.

Conflicts of Interest: The authors declare no conflicts of interest. The funders had no role in the design of the study; in the collection, analyses, or interpretation of data; in the writing of the manuscript; or in the decision to publish the results. 


\section{References}

1. D'Mello, M.J.J.; Ross, S.A.; Briel, M.; Anand, S.S.; Gerstein, H.; Paré, G. Association between shortened leukocyte telomere length and cardiometabolic outcomes: Systematic review and meta-analysis. Circ. Cardiovasc. Genet. 2015, 8, 82-90. [CrossRef] [PubMed]

2. Haycock, P.C.; Heydon, E.E.; Kaptoge, S.; Butterworth, A.S.; Thompson, A.; Willeit, P. Leucocyte telomere length and risk of cardiovascular disease: Systematic review and meta-analysis. BMJ 2014, 349, g4227. [CrossRef] [PubMed]

3. Codd, V.; Nelson, C.P.; Albrecht, E.; Mangino, M.; Deelen, J.; Buxton, J.L.; Hottenga, J.J.; Fischer, K.; Esko, T.; Surakka, I.; et al. Identification of seven loci affecting mean telomere length and their association with disease. Nat. Genet. 2013, 45, 422-427. [CrossRef] [PubMed]

4. Mangino, M.; Hwang, S.-J.; Spector, T.D.; Hunt, S.C.; Kimura, M.; Fitzpatrick, A.L.; Christiansen, L.; Petersen, I.; Elbers, C.C.; Harris, T.; et al. Genome-wide meta-analysis points to CTC1 and ZNF676 as genes regulating telomere homeostasis in humans. Hum. Mol. Genet. 2012, 21, 5385-5394. [CrossRef] [PubMed]

5. Haycock, P.C.; Burgess, S.; Nounu, A.; Zheng, J.; Okoli, G.N.; Bowden, J.; Wade, K.H.; Timpson, N.J.; Evans, D.M.; Willeit, P.; et al. Association Between Telomere Length and Risk of Cancer and Non-Neoplastic Diseases: A Mendelian Randomization Study. JAMA Oncol. 2017, 3, 636-651. [CrossRef] [PubMed]

6. Scheller Madrid, A.; Rode, L.; Nordestgaard, B.G.; Bojesen, S.E. Short Telomere Length and Ischemic Heart Disease: Observational and Genetic Studies in 290022 Individuals. Clin. Chem. 2016, 62, 1140-1149. [CrossRef] [PubMed]

7. Zhan, Y.; Karlsson, I.K.; Karlsson, R.; Tillander, A.; Reynolds, C.A.; Pedersen, N.L.; Hägg, S. Exploring the Causal Pathway From Telomere Length to Coronary Heart Disease: A Network Mendelian Randomization Study. Circ. Res. 2017, 121, 214-219. [CrossRef]

8. Benetos, A.; Toupance, S.; Gautier, S.; Labat, C.; Kimura, M.; Rossi, P.M.; Settembre, N.; Hubert, J.; Frimat, L.; Bertrand, B.; et al. Short Leukocyte Telomere Length Precedes Clinical Expression of Atherosclerosis: The Blood-and-Muscle Model. Circ. Res. 2018, 122, 616-623. [CrossRef]

9. Kimura, M.; Stone, R.C.; Hunt, S.C.; Skurnick, J.; Lu, X.; Cao, X.; Harley, C.B.; Aviv, A. Measurement of telomere length by the Southern blot analysis of terminal restriction fragment lengths. Nat. Protoc. 2010, 5 , 1596-1607. [CrossRef]

10. He, C.; Holme, J.; Anthony, J. SNP genotyping: The KASP assay. Methods Mol. Biol. 2014, 1145, 75-86. [CrossRef]

11. Sabharwal, S.; Verhulst, S.; Guirguis, G.; Kark, J.D.; Labat, C.; Roche, N.E.; Martimucci, K.; Patel, K.; Heller, D.S.; Kimura, M.; et al. Telomere length dynamics in early life: The blood-and-muscle model. FASEB J. 2018, 32, 529-534. [CrossRef] [PubMed]

12. Barraclough, J.Y.; Skilton, M.R.; Garden, F.L.; Toelle, B.G.; Marks, G.B.; Celermajer, D.S. Early and late childhood telomere length predict subclinical atherosclerosis at age 14 yrs.-The CardioCAPS study. Int. J. Cardiol. 2019, 278, 250-253. [CrossRef] [PubMed]

13. Skilton, M.R.; Nakhla, S.; Ayer, J.G.; Harmer, J.A.; Toelle, B.G.; Leeder, S.R.; Jones, G.; Marks, G.B.; Celermajer, D.S.; Childhood Asthma Prevention Study Group. Telomere length in early childhood: Early life risk factors and association with carotid intima-media thickness in later childhood. Eur. J. Prev. Cardiol. 2016, 23, 1086-1092. [CrossRef] [PubMed]

14. Toupance, S.; Labat, C.; Temmar, M.; Rossignol, P.; Kimura, M.; Aviv, A.; Benetos, A. Short Telomeres, but Not Telomere Attrition Rates, Are Associated With Carotid Atherosclerosis. Hypertension 2017, 70, 420-425. [CrossRef] [PubMed]

15. Chiriacò, M.; Georgiopoulos, G.; Duranti, E.; Antonioli, L.; Puxeddu, I.; Nannipieri, M.; Rosada, J.; Blandizzi, C.; Taddei, S.; Virdis, A.; et al. Inflammation and Vascular Ageing: From Telomeres to Novel Emerging Mechanisms. High Blood Press. Cardiovasc. Prev. Off. J. Ital. Soc. Hypertens. 2019, 26, 321-329. [CrossRef] [PubMed]

16. Kang, Y.; Zhang, H.; Zhao, Y.; Wang, Y.; Wang, W.; He, Y.; Zhang, W.; Zhang, W.; Zhu, X.; Zhou, Y.; et al. Telomere Dysfunction Disturbs Macrophage Mitochondrial Metabolism and the NLRP3 Inflammasome through the PGC-1 $\alpha$ /TNFAIP3 Axis. Cell Rep. 2018, 22, 3493-3506. [CrossRef]

17. Grebe, A.; Hoss, F.; Latz, E. NLRP3 Inflammasome and the IL-1 Pathway in Atherosclerosis. Circ. Res. 2018, 122, 1722-1740. [CrossRef] 
18. Amorós-Pérez, M.; Fuster, J.J. Clonal hematopoiesis driven by somatic mutations: A new player in atherosclerotic cardiovascular disease. Atherosclerosis 2020, 297, 120-126. [CrossRef]

19. Jaiswal, S.; Natarajan, P.; Silver, A.J.; Gibson, C.J.; Bick, A.G.; Shvartz, E.; McConkey, M.; Gupta, N.; Gabriel, S.; Ardissino, D.; et al. Clonal Hematopoiesis and Risk of Atherosclerotic Cardiovascular Disease. N. Engl. J. Med. 2017, 377, 111-121. [CrossRef]

20. Zink, F.; Stacey, S.N.; Norddahl, G.L.; Frigge, M.L.; Magnusson, O.T.; Jonsdottir, I.; Thorgeirsson, T.E.; Sigurdsson, A.; Gudjonsson, S.A.; Gudmundsson, J.; et al. Clonal hematopoiesis, with and without candidate driver mutations, is common in the elderly. Blood 2017, 130, 742-752. [CrossRef]

21. Schratz, K.E.; Haley, L.; Danoff, S.K.; Blackford, A.; DeZern, A.; Gocke, C.D.; Duffield, A.S.; Armanios, M. Cancer spectrum and outcomes in the Mendelian short telomere syndromes. Blood 2020. [CrossRef] [PubMed]

22. Aviv, A.; Levy, D. Hemothelium, Clonal Hematopoiesis of Indeterminate Potential, and Atherosclerosis. Circulation 2019, 139, 7-9. [CrossRef] [PubMed]

23. Steensma, D.P. Clinical Implications of Clonal Hematopoiesis. Mayo Clin. Proc. 2018, 93, 1122-1130. [CrossRef] [PubMed]

24. Lacaud, G.; Kouskoff, V. Hemangioblast, hemogenic endothelium, and primitive versus definitive hematopoiesis. Exp. Hematol. 2017, 49, 19-24. [CrossRef]

25. Aviv, A.; Levy, D. Telomeres, atherosclerosis, and the hemothelium: The longer view. Annu. Rev. Med. 2012, 63, 293-301. [CrossRef]

26. Aviv, A.; Hunt, S.C.; Lin, J.; Cao, X.; Kimura, M.; Blackburn, E. Impartial comparative analysis of measurement of leukocyte telomere length/DNA content by Southern blots and qPCR. Nucleic Acids Res. 2011, 39, e134. [CrossRef]

27. Nettle, D.; Seeker, L.; Nussey, D.; Froy, H.; Bateson, M. Consequences of measurement error in qPCR telomere data: A simulation study. PLoS ONE 2019, 14, e0216118. [CrossRef]

(C) 2020 by the authors. Licensee MDPI, Basel, Switzerland. This article is an open access article distributed under the terms and conditions of the Creative Commons Attribution (CC BY) license (http://creativecommons.org/licenses/by/4.0/). 